\title{
The role of the right hemisphere in the recovery of stroke-related aphasia: a systematic review
}

\author{
Elissa-Marie Cocquyt' ${ }^{1}$ Lisa De Ley', Patrick Santens², John Van Borsel'1, Miet De Letter ${ }^{1}$
}

\section{${ }^{1}$ Department of Speech, Language and Hearing Sciences, ${ }^{2}$ Department of Internal Medicine, Ghent University}

\section{Background}

Neuroplasticity is the key to aphasia recovery after stroke and may occur in both the left and right hemisphere. Language recovery, spontaneous or due to various therapeutic interventions, takes place in three phases (acute, subacute and chronic) and is influenced by time-, task-, injury-, language-, person- and therapy-related variables. Especially the role of the right hemisphere in language reorganization remains a matter of debate. Therefore, a systematic review on the existing literature was performed. The research questions were based on the PICOS-principle (Moher et al., 2009):

(1) "What is the role of the right hemisphere in the acute, subacute and chronic phase in spontaneous and therapy-related aphasia recovery?"

(2) "Which variables have an influence on the role of the right hemisphere?"

\section{Method}

$>$ Systematic review until October 15th, 2015

$>$ Electronic databases: Web of Science \& Pubmed

$>$ Two independent reviewers

$>$ Descriptive statistics

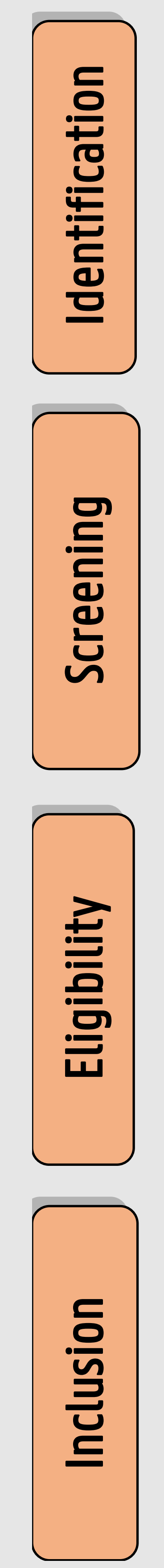

Records identified without duplicates $(n=1372)$

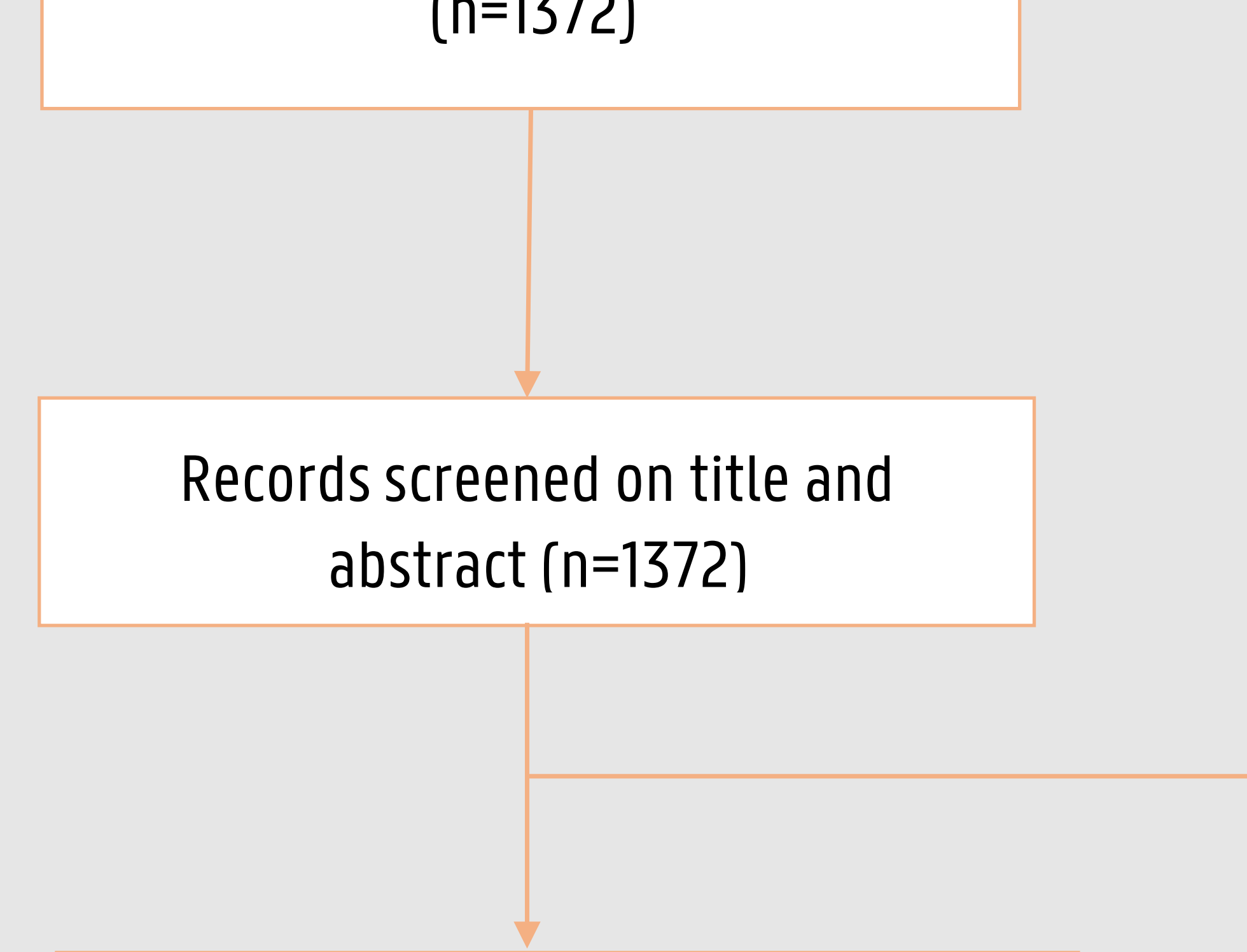

Records excluded $(n=1242)$

Full-text articles assessed for eligibility $(n=130)$

Figure: Flowchart of the study identification and selection process (Moher et al., 2009)

\section{References}

Cocquyt E.M., De Ley L., Santens P., Van Borsel J., De Letter M. (accepted). The role of the right hemisphere in the recovery of stroke-related aphasia: a systematic review. JNL. Moher D., Liberati A., Tetzlaff J., Altman D.G. (2009). Preferred Reporting Items for Systematic Reviews and Meta-Analyses:The PRISMA Statement. Ann Intern Med.

\section{Results}

Role of the right hemisphere in spontaneous aphasia recovery ( $n=5]$

\begin{tabular}{|c|c|c|c|}
\hline & Acute (n=1) & Subacute $(n=3)$ & Chronic ( $n=1$ ) \\
\hline $\begin{array}{c}\text { Auditory } \\
\text { comprehension }\end{array}$ & Facilitating (1/l]) & Facilitating (1/l]) & Unclear (1/1) \\
\hline $\begin{array}{c}\text { Reading } \\
\text { comprehension }\end{array}$ & & Facilitating (1/1) & \\
\hline Resting state & & No role $(1 / 1)$ & \\
\hline
\end{tabular}

Role of the right hemisphere in therapy-related aphasia recovery ( $n=48$ )

\begin{tabular}{|c|c|c|c|c|}
\hline & Acute (n=1) & Subacute $(n=3)$ & Chronic ( $n=44)$ & Follow-up $(n=8)$ \\
\hline $\begin{array}{c}\text { Auditory } \\
\text { comprehension }\end{array}$ & No role $(1 / 1)$ & $\begin{array}{c}\text { Facilitating }(1 / 2) \\
\text { Unclear }(1 / 2)\end{array}$ & $\begin{array}{c}\text { Facilitating }[5 / 9] \\
\text { No role }(2 / 9) \\
\text { Unclear }(2 / 9)\end{array}$ & $\begin{array}{l}\text { Inhibiting }(2 / 5) \\
\text { No role }(2 / 5) \\
\text { Unclear }(1 / 5)\end{array}$ \\
\hline $\begin{array}{c}\text { Reading } \\
\text { comprehension }\end{array}$ & & & $\begin{array}{l}\text { Facilitating }(1 / 2) \\
\text { Unclear }(1 / 2)\end{array}$ & \\
\hline Verbal production & & $\begin{array}{c}\text { Facilitating (1/2) } \\
\text { Unclear (1/2) }\end{array}$ & $\begin{array}{c}\text { Facilitating (14/22) } \\
\text { Inhibiting (2/22) } \\
\text { No role }(3 / 22) \\
\text { Unclear }(3 / 22)\end{array}$ & $\begin{array}{l}\text { Inhibiting }(1 / 3) \\
\text { Facilitating (2/3) }\end{array}$ \\
\hline Reading & & & $\begin{array}{c}\text { Facilitating }[3 / 6) \\
\text { No role }(1 / 6) \\
\text { Unclear }(2 / 6)\end{array}$ & \\
\hline Resting state & & & $\begin{array}{c}\text { Facilitating }(3 / 6) \\
\text { No role }(1 / 6) \\
\text { Unclear }(2 / 6)\end{array}$ & \\
\hline $\begin{array}{l}\text { Visual lexical } \\
\text { decision }\end{array}$ & & Facilitating (1/1) & Unclear (1/1) & \\
\hline
\end{tabular}

\section{Variables affecting the role of the right hemisphere $(n=11)$}

\begin{tabular}{|c|c|}
\hline & Influence on the right hemisphere \\
\hline $\begin{array}{l}\text { Lesion localization } \\
\qquad 5 / 11]\end{array}$ & $\begin{array}{l}\text { Subcortical lesions } \rightarrow \text { no role (spontaneous recovery) } \\
\text { Intact left basal ganlia } \rightarrow \text { facilitating role } \\
\text { Lesion of inferior frontal gyrus } \rightarrow \text { facilitating role }\end{array}$ \\
\hline $\begin{array}{c}\text { Aphasia severity } \\
\qquad(1 / 11)\end{array}$ & $\begin{array}{l}\text { Mild/moderate aphasia } \rightarrow \text { facilitating role } \\
\text { Severe aphasia } \rightarrow \text { no role }\end{array}$ \\
\hline $\begin{array}{c}\text { Early start, therapy } \\
\text { intensity \& content } \\
\text { (5/11] }\end{array}$ & $\begin{array}{l}\text { Early therapy start } \rightarrow \text { no role } \\
\text { Intensive therapy } \rightarrow \text { facilitating role } \\
\text { Melodic Intonation Therapy, Phonological therapy } \rightarrow \text { facilitating role }\end{array}$ \\
\hline
\end{tabular}

\section{Conclusion}

The right hemisphere might contribute to early spontaneous recovery in the acute and subacute recovery phase. In chronic aphasia, the right hemisphere initially facilitates therapyrelated recovery but does not support, or even inhibits, recovery in the longer term. Nevertheless, more systematic follow-up studies and further research on variables affecting the role of the right hemisphere in aphasia recovery are required. 